\title{
A non-invasive node-based form finding approach with discretization-independent target configuration
}

\author{
Michael Caspari@*, Philipp Landkammer and Paul Steinmann
}

\author{
${ }^{*}$ Correspondence: \\ michael.caspari@fau.de \\ Institute of Applied Mechanics, \\ Friedrich Alexander Universität, \\ Egerlandstraße 5, 91058 \\ Erlangen, Germany
}

\begin{abstract}
Form finding is used to optimize the shape of a semi-finished product, i.e. the material configuration in a forming process. The geometry of the semi-finished product is adapted so that the computed spatial configuration corresponds to a prescribed target spatial configuration. Differences between these two configurations are iteratively minimized. The algorithm works non-invasively, thus there is a strict separation between the form update and the finite element (FE) forming simulation. This separation allows the use of arbitrary commercial FE-solvers. In particular, there is no need for a modification of the FE forming simulation, only the material configuration is iteratively updated. A new method is introduced to calculate the difference between the target and the computed spatial configuration. Thereby the target mesh is separated from the mesh for the FE forming simulation, which enables a more accurate and independent representation of the target configuration. In addition, the possibility of taking into account manufacturing constraints in the optimization process is presented. The procedure is illustrated for the example of the first stage of a novel two-stage sheet-bulk metal forming process.
\end{abstract}

Keywords: Form finding, Shape optimization, Metal forming, Non-invasive methods, Inverse problems

\section{Introduction}

Metal forming processes are distinguished into sheet and bulk metal forming. This classification results from the prevailing stress state of the respective forming operation. Sheet metal forming is characterized by a two-dimensional stress state, whereas for bulk metal forming, three-dimensional stress states dominate. The recent sheet-bulk metal forming (SBMF) process, presented in [1], combines these two basic processes into a single more complex process. The underlying idea is to integrate individual functional elements, manufactured by bulk forming, into a component created by sheet forming. This novel technology is currently investigated with much rigor in a DFG ${ }^{1}$-funded collaborative research project: Manufacturing of complex functional components with variants by using a new sheet metal forming process-Sheet-Bulk Metal Forming (TR73). Within this project in particular tailored blanks are studied, i.e. semi-finished products are a priorly adapted

\footnotetext{
${ }^{1}$ DFG is the self-governing organisation for science and research in Germany (http://www.dfg.de).
}

(c) The Author(s) 2018. This article is distributed under the terms of the Creative Commons Attribution 4.0 International License (http://creativecommons.org/licenses/by/4.0/), which permits unrestricted use, distribution, and reproduction in any medium, provided you give appropriate credit to the original author(s) and the source, provide a link to the Creative Commons license, and indicate if changes were made. 
to the intended SBMF process. For this purpose, processes for material pre-distribution are used, see [2].

In this contribution we focus as an example on a two-stage forming process from the family of SBMF processes. This process is used to illustrate the procedure of form finding while demonstrating improvements in optimizing more complex geometries and taking into account manufacturing constraints. In general a direct problem is distinguished from an inverse problem, see Chenot et al. [3]. A forming simulation is a direct problem where the material configuration is given and the spatial configuration is sought. If the spatial configuration is prescribed and the material configuration is sought, it represents an inverse problem. According to this classification, illustrated in Fig. 1, form finding in the sense of shape optimization is an inverse problem.

Figure 1 illustrates furthermore the concept of the proposed non-invasive form finding. Accordingly, the optimization cycle starts with an initial material configuration (left, black color) as input for the FE forming simulation. The FE forming simulation itself is carried out independently of the optimization and computes the spatial configuration (right, black color) as a final result. This computed spatial configuration is compared with a target spatial configuration and a suitably defined spatial difference vector is calculated. The algorithm projects the spatial difference vector to the material configuration for the form update. The procedure iteratively minimizes the spatial difference vector. In addition, the algorithm works on a node-based basis, so that each FE node is taken into account individually for optimization.

The optimization loop is used to determine an optimal semi-finished product geometry. The resulting optimized geometry ensures that the desired spatial configuration is achieved for given loading and boundary conditions. During optimization, the FE-solver for the forming simulation is not affected, indeed it is entirely independent from the form finding, thus it is in particular possible to incorporate arbitrary commercial FE-solvers, which is a substantial asset for the industrial application of the algorithm.

The basic approach was first introduced by Landkammer and Steinmann [4]. Various enhancements to improve the algorithm in terms of both accuracy and versatility have

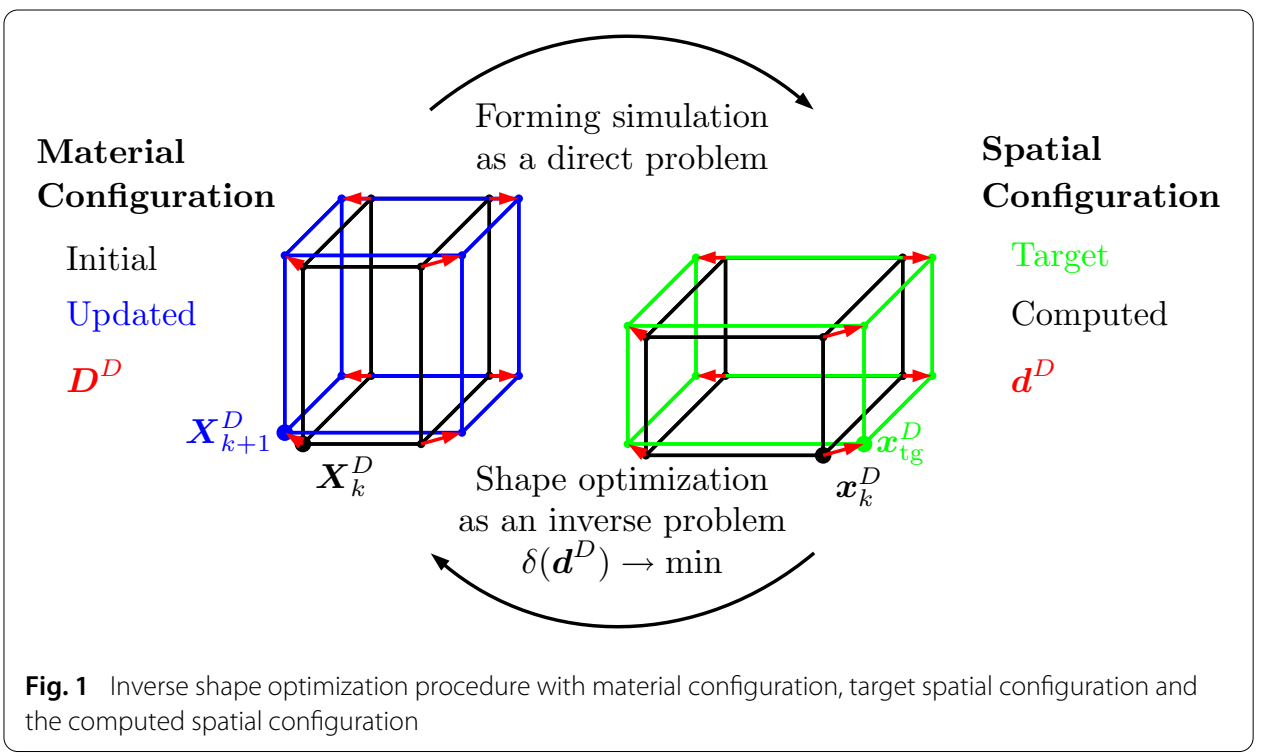


been presented in $[5,6]$. The focus of our current work is on the further improvement of its stability and robustness. The following developments include two major modifications of the optimization algorithm in order to enhance its applicability to real processes. Recent investigations emphasized a strong dependency of the optimized material position of individual nodes on the respective nodes in the target spatial configuration. This dependency is entirely bypassed by releasing the mesh of the target spatial configuration from that of the FE forming simulation ("Detachment of the target mesh from the mesh of the forming simulation" section). This does not change the type of optimization, but the way of computing the differences between the computed spatial configuration and the target spatial configuration. In addition, a first step is made to consider manufacturability ("Constraining the available design space" section) of the optimization result when manufacturing constraints must be taken into account. The constraint considered here is the available design space for the semi-finished product, which is limited by the material pre-distribution process.

In the following, the basic principles of nonlinear continuum mechanics are briefly re-iterated ("Basics of nonlinear continuum mechanics" section). This is followed by the description of the update step for a single node ("Description of an update step" section). The entire algorithm is outlined afterwards. Here, the above-mentioned improvements will also be presented. Finally, the procedure is illustrated by an example in "A two-stage forming process of a tailored blank" section. The last chapter summarizes the findings.

\section{Basics of nonlinear continuum mechanics}

Basics of nonlinear continuum mechanics are reiterated as a preliminary to the derivation of the subsequent algorithm for form finding, whereby the following presentation is limited to some important assumptions. A more detailed description is represented in $[7,8]$.

\section{Kinematics of the continuous setting}

In continuum mechanics, a distinction is made between two configurations of a continuous body: the material configuration $\mathcal{B}_{0}$ at time $t=0$ and the spatial configuration $\mathcal{B}_{t}$ at time $t>0$. Assuming an Euclidean space $\mathbb{E}^{3}$ with basis vectors $\boldsymbol{E}_{i} \equiv \boldsymbol{e}_{i}$ and $i=1,2,3$, these two configurations are represented as illustrated in Fig. 2.

The kinematic relationships between the two configurations include the deformation map, the displacement field and the deformation gradient. The deformation map $\varphi$ assigns to a position vector $X$ in the material (undeformed) configuration $\mathcal{B}_{0}$ a position vector $\boldsymbol{x}$ in the spatial (deformed) configuration $\mathcal{B}_{t}$ :

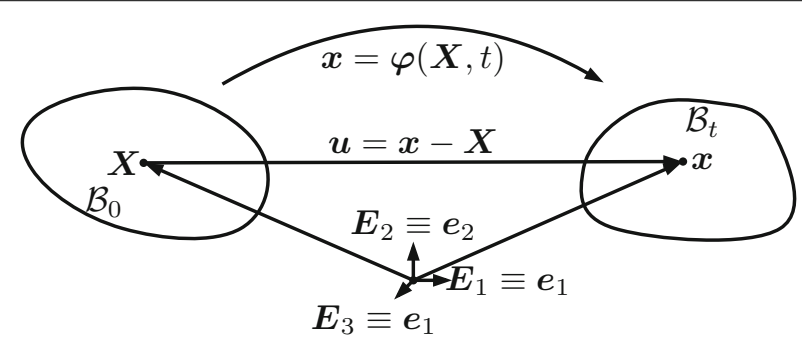

Fig. 2 Material (undeformed) and the spatial (deformed) configuration of a continuous body 


$$
\boldsymbol{x}=\varphi(X, t) \quad: \mathcal{B}_{0} \rightarrow \mathcal{B}_{t}
$$

The displacement field $\boldsymbol{u}$ results from the difference of the position vectors in the spatial and the material configuration:

$$
\boldsymbol{u}(X, t)=\varphi(X, t)-X
$$

The gradient $\boldsymbol{F}$ of the deformation map with respect to the material coordinates renders a linear map from the material tangent space $T \mathcal{B}_{0}$ to the spatial tangent space $T \mathcal{B}_{t}$ :

$$
\boldsymbol{F}=\frac{\partial \varphi(X)}{\partial X} \quad: T \mathcal{B}_{0} \rightarrow T \mathcal{B}_{t}
$$

\section{Weak form of balance of forces}

Due to the dominance of contact forces involved in metal forming processes, body forces are here neglected without loss of generality. Equilibrium is formulated as a boundary value problem:

$$
\operatorname{Div} \boldsymbol{P}=\mathbf{0},
$$

whereby the corresponding boundary conditions decompose the Dirichlet and Neumann conditions:

$$
\boldsymbol{\varphi}=\overline{\boldsymbol{\varphi}} \quad \text { on } \quad \partial \mathcal{B}_{0}^{\varphi} \quad \text { and } \quad \boldsymbol{P} \cdot \boldsymbol{N}=\boldsymbol{T} \quad \text { on } \quad \partial \mathcal{B}_{0}^{T}
$$

The equilibrium equation (Eq. 4) is formulated in terms of the Piola stress $\boldsymbol{P}$ and results from the balance of forces.

These equations yield a system of PDEs, that is solved based on the principle of virtual power. To this end, virtual displacements $\delta \varphi$ are introduced. The weak form incorporating the boundary conditions is formulated as follows:

$$
\int_{\mathcal{B}_{0}} \boldsymbol{P}: \delta \boldsymbol{F} \mathrm{d} V=\int_{\partial \mathcal{B}_{0}^{T}} \delta \boldsymbol{\varphi} \cdot \boldsymbol{T} \mathrm{d} A \quad \forall \delta \boldsymbol{\varphi} \text { admissible. }
$$

\section{Solving the weak form by applying a FE discretization}

In order to solve the weak form in Eq. 6, it is necessary to discretize the continuous body $\mathcal{B}$ into finite elements.

In the following the same discretization is used for both, the coordinates and the field values. Within the finite element computations, integrals are typically computed by numerical integration, i.e. Gauss quadrature. Finally, efficient iterative solution methods are used to solve the resulting system of nonlinear algebraic equations. For a detailed account on the FE-method, we refer to [9], among others.

The body $\mathcal{B}$ is discretized into $n_{\text {elem }}$ elements:

$$
\mathcal{B}_{0} \approx \mathcal{B}_{0}^{\mathrm{h}}=\bigcup_{e=1}^{n_{\text {elem }}} \mathcal{B}_{0}^{e} \quad \text { and } \quad \mathcal{B}_{t} \approx \mathcal{B}_{t}^{\mathrm{h}}=\bigcup_{e=1}^{n_{\text {elem }}} \mathcal{B}_{t}^{e} .
$$

Likewise the coordinates in the material and spatial configuration are discretized as:

$$
\boldsymbol{X} \approx \boldsymbol{X}^{\mathrm{h}}=\bigcup_{e=1}^{n_{\text {elem }}} \boldsymbol{X}^{e} \quad \text { and } \quad \boldsymbol{x} \approx \boldsymbol{x}^{\mathrm{h}}=\bigcup_{e=1}^{n_{\text {elem }}} \boldsymbol{x}^{e} .
$$


Within the isoparametric concept, all kinematic quantities are approximated by the same shape functions $N^{i}(\xi)$ for each element node $\left(i=1 \ldots n_{\mathrm{en}}\right)$, which are defined on a reference element $\mathcal{B}_{\square}$ with isoparametric coordinates $\xi \in[-1,1]^{n_{\text {dim }}}$. The coordinates $\boldsymbol{X}^{e}$ and $\boldsymbol{x}^{e}$ within an element depend on the nodal positions $\boldsymbol{X}^{i}$ and $\boldsymbol{x}^{i}$ :

$$
\boldsymbol{X}^{e}(\xi)=\sum_{i=1}^{n_{\mathrm{en}}} \boldsymbol{X}^{i} N^{i}(\xi) \quad \text { and } \quad \boldsymbol{x}^{e}(\xi)=\sum_{i=1}^{n_{\mathrm{en}}} \boldsymbol{x}^{i} N^{i}(\xi)
$$

The discretized deformation map and deformation gradient follow as:

$$
x^{\mathrm{h}}=\varphi\left(X^{\mathrm{h}}, t\right) \quad: \mathcal{B}_{0}^{\mathrm{h}} \rightarrow \mathcal{B}_{t}^{\mathrm{h}} \quad \text { and } \quad F^{\mathrm{h}}=\frac{\partial \varphi\left(X^{\mathrm{h}}\right)}{\partial X^{\mathrm{h}}} \quad: T \mathcal{B}_{0}^{\mathrm{h}} \rightarrow T \mathcal{B}_{t}^{\mathrm{h}} .
$$

The material and spatial Jacobian

$$
\boldsymbol{J}^{e}(\boldsymbol{\xi})=\sum_{i=1}^{n_{\mathrm{en}}} \boldsymbol{X}^{i} \otimes \frac{\partial N^{i}(\boldsymbol{\xi})}{\partial \boldsymbol{\xi}} \quad \text { and } \quad \boldsymbol{j}^{e}(\boldsymbol{\xi})=\sum_{i=1}^{n_{\mathrm{en}}} \boldsymbol{x}^{i} \otimes \frac{\partial N^{i}(\boldsymbol{\xi})}{\partial \boldsymbol{\xi}}
$$

are used for the mapping from the reference element to the element in the material or the spatial configuration, respectively, the deformation gradient $\boldsymbol{F}^{e}$ is thus evaluated by:

$$
\boldsymbol{F}^{e}(\boldsymbol{\xi})=\boldsymbol{j}^{e}(\boldsymbol{\xi}) \cdot \boldsymbol{J}^{e}(\boldsymbol{\xi})^{-1}=\left[\sum_{i=1}^{n_{\mathrm{en}}} \boldsymbol{x}^{i} \otimes \frac{\partial N^{i}(\boldsymbol{\xi})}{\partial \boldsymbol{\xi}}\right] \cdot\left[\sum_{i=1}^{n_{\mathrm{en}}} \boldsymbol{X}^{i} \otimes \frac{\partial N^{i}(\boldsymbol{\xi})}{\partial \boldsymbol{\xi}}\right]^{-1}
$$

Figure 3 show cases a finite element in the material, spatial and reference configuration, respectively.

\section{Description of an update step}

The proposed optimization approach is based on the following concept:

Each discretization node on the external boundary of the continuous body has an optimal material position, which leads to the target spatial configuration after applying the forming simulation.

The objective of the optimization is to determine this optimal material position by means of an iteratively repeated optimization step. The mathematical derivation of a single update step is explained below.

The difference between the current spatial position of a design node $x^{D}=\varphi\left(X^{D}\right)$ with global node index $D$ and its target spatial position $x_{\text {tg }}^{D}$ defines the spatial difference vector or rather the error of the nodal spatial position:

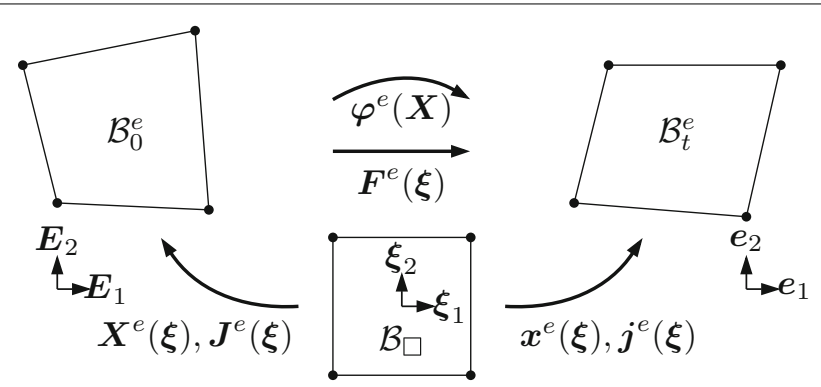

Fig. 3 Discretized setting with the reference element $\mathcal{B}_{\square}$, material element $\mathcal{B}_{0}^{e}$ and the spatial element $\mathcal{B}_{t}^{e}$ 


$$
d^{D}=x_{\mathrm{tg}}^{D}-\varphi\left(X^{D}\right)
$$

These differences, calculated for each design node $D$, are merged into a global objective function:

$$
\delta\left(\mathbf{X}^{\mathrm{D}}, \mathbf{x}_{\mathrm{tg}}^{\mathrm{D}}\right)=\sum_{D=1}^{n_{\mathrm{dsgn}}} \delta^{D}\left(\boldsymbol{x}_{\mathrm{tg}}^{D}, \boldsymbol{\varphi}\left(\boldsymbol{X}^{D}\right)\right)
$$

where by $\delta^{D}$ is denoted the nodal objective function defined by the squared error of a nodal spatial position:

$$
\delta^{D}=\frac{1}{2} \boldsymbol{d}^{D^{\top}} \cdot \boldsymbol{d}^{D}
$$

Design nodes contain at least one design coordinate and are located on the external boundary of the geometry. The global objective function contains only the differences of design coordinates. Further coordinates are fixed coordinates, which are restricted by boundary conditions and are therefore not relocated during the update step and the controlled coordinates which are updated by a fictitious elastic problem. Nodes on the external boundary can have both design and fixed coordinates, whereas nodes in the interior of the geometry only contain controlled coordinates. This kind of distinction is commonly used for parameter free (node-based) optimization procedures and is outlined in more detail in [10].

The column vectors on the left hand side of Eq. $14, \mathbf{X}^{\mathrm{D}}$ and $\mathbf{x}^{\mathrm{D}}$, store all material and spatial positions of the $n_{\mathrm{dsgn}}$ design nodes. The optimization approach is summarized in Table 1.

For determining the optimal material positions $\mathbf{X}_{\mathrm{opt}}^{\mathrm{D}}$ of all design nodes (and so the corresponding optimal material configuration) the global objective function is minimized with respect to the material coordinates. Thus, the optimal material configuration $\mathbf{X}_{\mathrm{opt}}^{\mathrm{D}}$ has to satisfy the stationarity condition:

$$
\left.\frac{\partial \delta\left(\mathbf{X}^{\mathrm{D}}, \mathbf{x}_{\mathrm{tg}}^{\mathrm{D}}\right)}{\partial \mathbf{X}^{\mathrm{D}}}\right|_{\mathbf{X}_{\mathrm{opt}}^{\mathrm{D}}} \stackrel{!}{=} \mathbf{0} .
$$

The iterative update step follows from the approximation of Eq. 16 using a Taylor expansion truncated after the first term. This results in an iteration step as:

$$
\mathbf{X}_{k+1}^{\mathrm{D}}=\mathbf{X}_{k}^{\mathrm{D}}-\frac{\partial^{2} \delta\left(\mathbf{X}^{\mathrm{D}}, \mathbf{x}_{\mathrm{tg}}^{\mathrm{D}}\right)^{-1}}{\partial \mathbf{X}^{\mathrm{D}} \partial \mathbf{X}^{\mathrm{D}}} \cdot \frac{\partial \delta\left(\mathbf{X}^{\mathrm{D}}, \mathbf{x}_{\mathrm{tg}}^{\mathrm{D}}\right)}{\partial \mathbf{X}^{\mathrm{D}}}
$$

Equation 17 represents a global treatment, however we decide to only locally perform the update step of the optimization procedure, so that the new material position of each

Table 1 The node-based optimization problem for inverse form finding [5]

\begin{tabular}{ll}
\hline Objective function & $\delta\left(\mathbf{X}^{\mathrm{D}}, \mathbf{x}_{\mathrm{tg}}^{\mathrm{D}}\right)=\sum_{D=1}^{n_{\mathrm{dsgn}}} \frac{1}{2}\left\|\boldsymbol{d}^{D}\right\|_{2}^{2}$ \\
Design variables & Material positions $\boldsymbol{X}^{\mathrm{D}}$ of the design nodes \\
State equation & Deformation map $\boldsymbol{\varphi}: \mathbf{X}^{\mathrm{D}} \in \mathcal{B}_{0}^{\mathrm{h}} \rightarrow \mathbf{x}^{\mathrm{D}} \in \mathcal{B}_{t}^{\mathrm{h}}$ \\
\hline
\end{tabular}


design node is calculated individually. The update step for each design node is thus written as (note the different fonts for the global $\mathbf{X}^{D}$ and the node-wise $\boldsymbol{X}^{D}$ ):

$$
\boldsymbol{X}_{k+1}^{D}=\boldsymbol{X}_{k}^{D}-\alpha \frac{\partial^{2} \delta^{D}\left(\boldsymbol{X}^{D}, \boldsymbol{x}_{\mathrm{tg}}^{\mathrm{h}}\right)}{\partial \boldsymbol{X}^{D} \partial \boldsymbol{X}^{D}} \cdot \frac{\partial \delta^{D}\left(\boldsymbol{X}^{D}, \boldsymbol{x}_{\mathrm{tg}}^{\mathrm{h}}\right)}{\partial \boldsymbol{X}^{D}} .
$$

The update step is necessarily complemented by a linesearch parameter $\alpha$ which is controlled by Armijo-Backtracking [5] and that ensures a suited update without severe distortion of a single element.

Equation 18 includes the first derivative (Jacobian) and the inverse of the second derivative (Hessian), $\boldsymbol{J}_{k}^{D}=\frac{\partial \delta^{D}\left(\boldsymbol{X}^{D}, \boldsymbol{x}_{\mathrm{tg}}^{\mathrm{h}}\right)}{\partial \boldsymbol{X}^{D}}=-\boldsymbol{F}_{\boldsymbol{k}}^{D^{\top}} \cdot \boldsymbol{d}_{\boldsymbol{k}}^{D}$ and $\boldsymbol{H}_{\boldsymbol{k}}^{D}=\frac{\partial^{2} \delta^{D}\left(\boldsymbol{X}^{D}, \boldsymbol{x}_{\mathrm{g}}^{\mathrm{h}}\right)}{\partial \boldsymbol{X}^{D} \partial \boldsymbol{X}^{D}}$, respectively, of the local objective function $\delta^{D}$ with respect to the nodal material position $\boldsymbol{X}^{D}$. Further transformations result in the update step for the $k$-th iteration:

$$
\boldsymbol{X}_{k+1}^{D}=\boldsymbol{X}_{k}^{D}+\alpha \tilde{\boldsymbol{H}}_{k}^{D^{-1}} \cdot \tilde{\boldsymbol{F}}_{k}^{D^{\top}} \cdot \boldsymbol{d}_{k}^{D}
$$

Here, the first deformation gradient and the Hessian are represented as smoothed quantities $\tilde{\boldsymbol{F}}$ and $\tilde{\boldsymbol{H}}$. According to Eq. 12, the gradients are calculated based on the ansatzfunctions evaluated at the Gauss points. However, the update requires values at the design nodes, so that smoothing is required. The values at the Gauss points of the adjoining elements of a design node are smoothed to the corresponding nodal value. Various possibilities for this procedure were also examined in [5]. As suggested by a detailed analysis, the superconvergent patch recovery technique as proposed in [11] is eventually used.

Equation 19 is further converted by approximation of the Hessian matrix. Different Quasi-Newton methods, outlined in [5], serve this purpose. Therein, numerical investigations suggest that the use of the Gauss-Newton approximation works most efficient and sufficiently accurate. With the Gauss-Newton approximation the update step finally reads as:

$$
\boldsymbol{X}_{k+1}^{D}=\boldsymbol{X}_{k}^{D}+\alpha \tilde{\boldsymbol{F}}_{k}^{D^{-1}} \cdot \boldsymbol{d}_{k}^{D}
$$

\section{A non-invasive form finding approach}

The special feature of the proposed non-invasive approach is the separation of the forming simulation and the optimization of the material configuration. It enables us to use arbitrary FE-solvers and to apply the optimization approach entirely independent. All required information is transferred by means of subroutines between the FE-solver and the optimization tool. At the end of the update step the new material configuration $\mathcal{B}_{0 k+1}$ is transferred back to the FE-solver. Finally, the forming simulation is restarted. The only information to be exchanged are the material positions of the design, fixed, and controlled nodes, other quantities such as plastic strains and stresses are not exchanged. If by way of example Abaqus is applied as FE-solver, Python and Fortran scripts are used for data exchange, for Marc/Mentat subroutines are usually Fortran as well. The data are transferred in text files individually adapted to the solver. In order to enable the use of additional solvers, the possibilities for data exchange of the respective solver must be taken into consideration. Once the updated material configuration $X_{k+1}$ is passed to the solver, the FE-problem is solved without any interference of the optimization algorithm. This kind of separation is denoted the non-invasive approach. 
As already mentioned, we distinguish between design, fixed and controlled nodal coordinates. Fixed coordinates belong to a node that is restricted by a Dirichlet boundary condition and therefore remain unchanged during the optimization. The design coordinates are updated by Eq. 20. Independently updating the controlled nodes is firstly proposed in [5]. The update of the design coordinates is specified by the nodal material difference vector $\boldsymbol{D}_{k}^{D}=\alpha \tilde{\boldsymbol{F}}_{k}^{D^{-1}} \cdot \boldsymbol{d}_{k}^{D}$ (with $D=1, \ldots, n_{\mathrm{dsgn}}$ ) for the $D$ th design node and $k$ th iteration. These nodal material difference vectors are used as prescribed Dirichlet boundary data of a fictitious elastic problem, which is solved once again with the selected FE-solver. The prescribed displacement of the design nodes lead to a relocation and thus update the material positions of the controlled nodes. The fictitious elastic problem has no mechanical relevance regarding the deformation of the material, it is only used to update the controlled coordinates of the FE-mesh to the previously determined relocation of the design coordinates. The non-invasive approach with the outlined procedure is pictured in Fig. 4.

Remark Note that the simulation of the forming process is much more time consuming than the computation of an updated material configuration. For the later introduced example, which is illustrated in Fig. 11b, in combination with the target mesh in Fig. 13b, the computational time decomposes into:

- Structure analysis $305 \mathrm{~s}$.

- Elastic update $4 \mathrm{~s}$.

- Update computation $10 \mathrm{~s}$.

Therefore, the computational cost of the update calculation is negligible and efficiency of the algorithm is not an issue and therefore not considered in detail.

\section{Detachment of the target mesh from the mesh of the forming simulation}

The update step outlined in "Description of an update step" section is determined by the nodal differences $\boldsymbol{d}^{D}$ between the target spatial position of the particular design node $\boldsymbol{x}_{\mathrm{tg}}^{D}$ and its computed spatial position $\varphi\left(X^{D}\right)$. A challenge arises from the dependence of the spatial position of a design node on its target spatial position that restricts the final material position of the design node. This problem becomes obvious by comparing the optimal material configuration of a simulation of the notch stamping process, outlined in $[6,12]$, for using two differently discretized target spatial configurations.

The target configurations to which the respective optimization was aimed are depicted in Fig. 5. It turns out to have a significant influence on the optimal material shape whether the target discretizations Fig. $5 \mathrm{a}$ or $\mathrm{b}$ is used. Figure 6 shows the setting of a structural

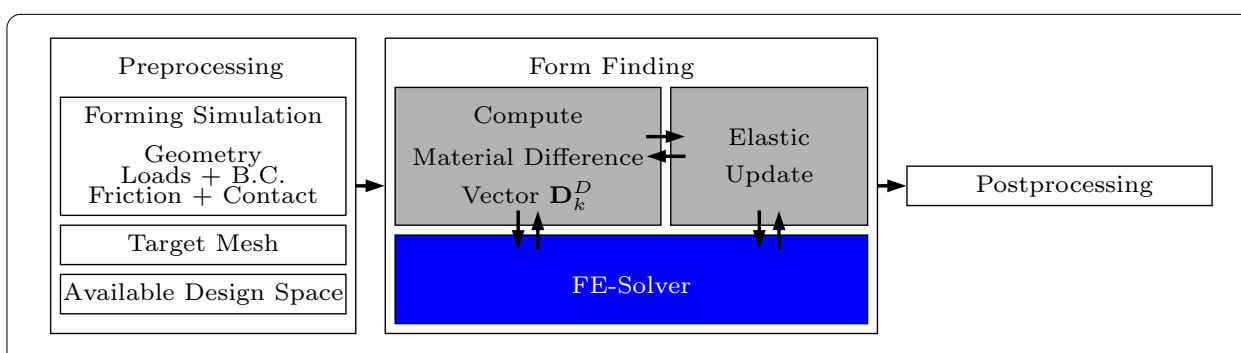

Fig. 4 Schematic representation of the optimization procedure 


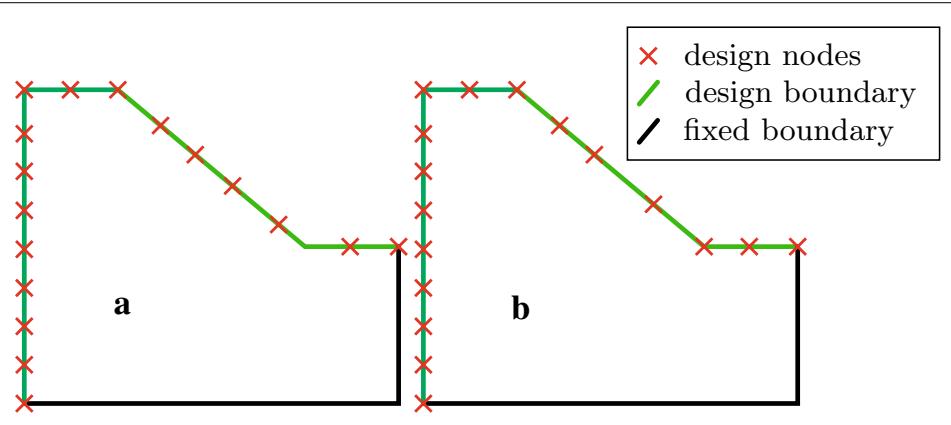

Fig. 5 Two identical target shapes with differing positions of the design nodes. Configuration a as used in [6] and $\mathbf{b}$ with two modified nodal positions

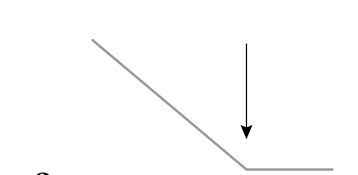

a

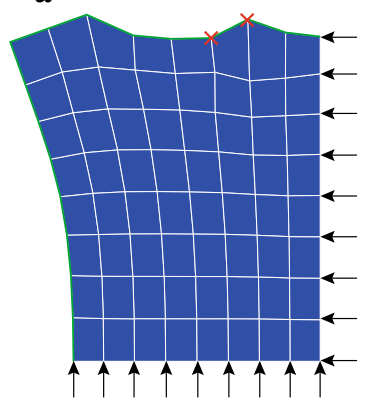

b

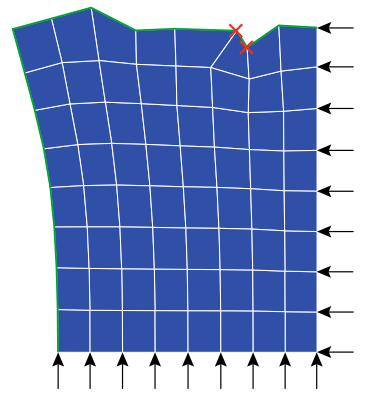

Fig. 6 Structural analysis setting with two optimized material configurations. Configuration a is based on the discretized target configuration in Fig. 5a. Configuration $\mathbf{b}$ is based on the discretized target configuration in Fig. 5b

analysis with two optimized material configurations. Although both optimizations are based on the target shape, the results differ for the two marked design nodes. This effect occurs despite of both target discretizations render the same shape but varied nodal positions.

The mismatch goes back to the computation of the difference vectors with its dependency on the nodal target positions. The aim is to introduce a novel way of computing the difference vector independently from the position of one particular target node. Indeed, the computation of the update for each design node has only to dependent on the geometry of the target. This offers the opportunity to design a target mesh with a different number of nodes and connectivity compared to that of the forming simulation. The target geometry is discretized by an independent FE-mesh with the positions $\boldsymbol{x}_{\mathrm{tg}-\mathrm{sf}}^{j}\left(\right.$ for $\left.j=1, \ldots, n_{\mathrm{tg}-\mathrm{sf}}\right)$ for its surface nodes.

The computation of the difference vector is performed in three steps.

\section{Projection step}

First, the projection step, outlined in Fig. 7b, performs a projection:

$$
\text { spatial nodes } x_{k}^{i} \rightarrow \text { surface of the target configuration }
$$




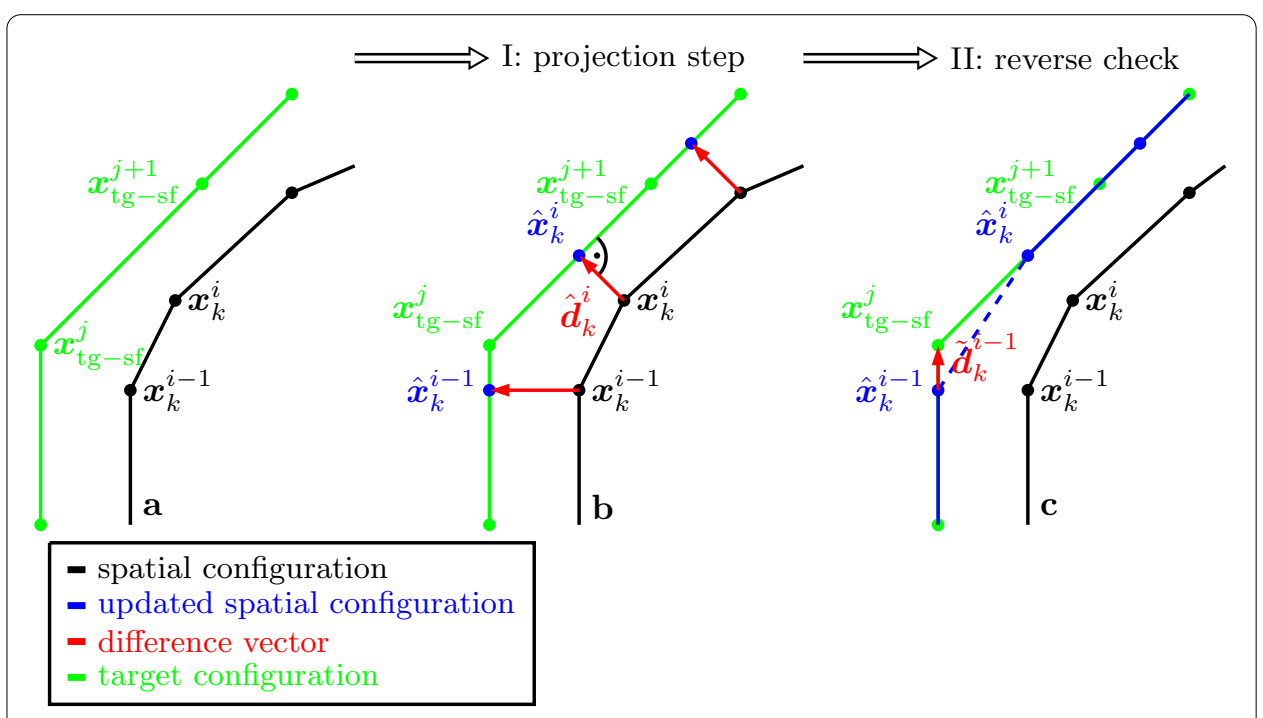

Fig. 7 (a) Starting scenario of the comparison between spatial and target configuraiton. Computation of the difference vector $\hat{\boldsymbol{d}}_{k}^{i}$ in the projection step (b) and the difference vector $\tilde{\boldsymbol{d}}_{k}^{i-1}$ for the reverse check (c)

A design node of the spatial configuration $x_{k}^{i}$ is projected onto the target mesh. Therefore, the two closest target design nodes are determined. The line between these two nodes is part of the target surface. For the difference vector $\hat{\boldsymbol{d}}_{k}^{i}$ of the $i$-th design node the orthogonal projection onto this line is computed as described in Algorithm 1.

\section{Reverse check}

Second, the reverse check projects:

target surface node $x_{\mathrm{tg}-\mathrm{sf}}^{j} \rightarrow$ updated spatial design surface

Data: Positions of spatial deformed design nodes $\boldsymbol{x}^{i}$ (for $i=1, \ldots, n_{\mathrm{dsgn}}$ ), Positions of spatial target design nodes $\boldsymbol{x}_{\mathrm{tg}}^{j}$ (for $\left.i=j, \ldots, n_{\mathrm{tg}-\mathrm{sf}}\right)$

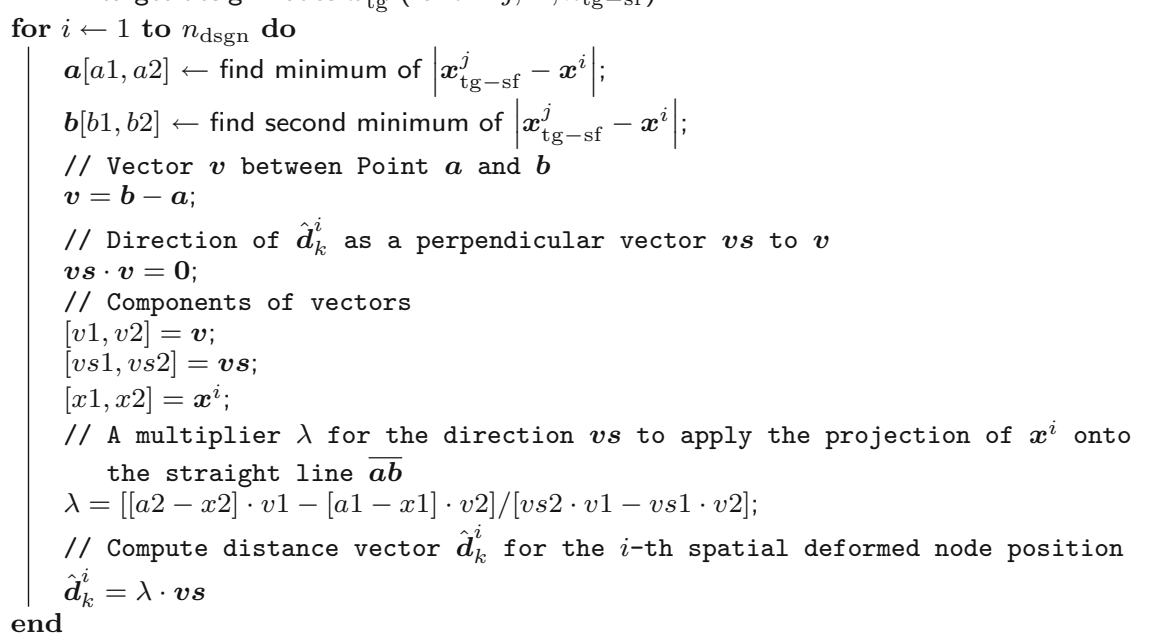

Result: Difference vector $\hat{\boldsymbol{d}}_{k}^{i}$ (for $i=1, \ldots, n_{\mathrm{dsgn}}$ ) for the projection step

Algorithm 1: Computation of the difference vector $\hat{\boldsymbol{d}}_{k}^{i}$ within a two-dimensional setting 
By using the computed difference vector $\hat{\boldsymbol{d}}_{k}^{i}$ it is ensured that the updated spatial configuration fits to the target surface. However, it is not ensured that every node of the target surface, especially nodes corresponding to a corner, fit to the updated shape of the spatial configuration. This case is illustrated in Fig. $7 \mathrm{~b}$ for the nodal positions $x_{\mathrm{tg}-\mathrm{sf}}^{j}$ of the target configuration. The reverse check is performed by using the updated spatial geometry from the projection step and calculating the projection of every target node $x_{\text {tg-sf }}^{j}$ (for $j=1 \ldots n_{\mathrm{tg}-\mathrm{sf}}$ ) onto this updated geometry.

The projection is performed similarly to the projection in Algorithm 1. However, the target nodes are projected reverse onto the updated spatial configuration. If the magnitude of the projection is bigger than a certain threshold, the difference vector for the reverse step $\tilde{\boldsymbol{d}}_{k}^{i}$ is computed by using the closest updated design node $\hat{\boldsymbol{x}}_{k}^{i}$ and calculating the difference accordingly. Eventually, the used closest spatial design node is updated again.

\section{III. $90^{\circ}$-corner check}

The last part is called the $\mathbf{9 0 ^ { \circ }}$-corner check. Within the first two steps a rectangular projection between one node and a straight line between two other nodes is performed. Figure 8 shows a special case of an updated spatial design node which does not fit to the target shape despite the projection- and reverse step being already performed. In the case of a rectangular projection onto a straight line corresponding to a $90^{\circ}$-corner, the update is placed close to the corner, however not onto the corner. Also the reverse check is not able to identify the improper update since the corner is part of the updated geometry. For this case all $90^{\circ}$-corners of the target shape are identified by a third update whereby $\overline{\boldsymbol{d}}_{k}^{D}$ is calculated for the node closest to the corner.

At the end, the final difference vector for each spatial position of a design node for the $k$-th iteration step is composed by summation:

$$
\boldsymbol{d}_{k}^{D}=\overline{\boldsymbol{d}}_{k}^{D}+\hat{\boldsymbol{d}}_{k}^{D}+\tilde{\boldsymbol{d}}_{k}^{D}
$$

\section{Update of the material configuration with a limited available design space}

Shape optimization often results in a contradiction between a theoretically optimal material configuration and manufacturing constraints. The updated material configuration is computed with Eq. 20, whereby the material difference vector is identified as:

$$
\boldsymbol{D}_{k}^{D}=\alpha \tilde{\boldsymbol{F}}_{k}^{D^{-1}} \cdot \boldsymbol{d}_{k}^{D}
$$

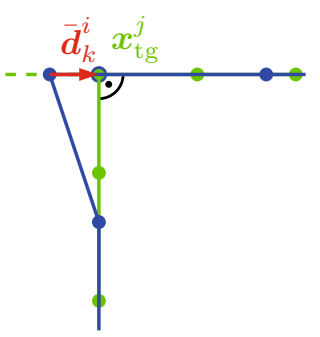

- updated spatial configuration

- difference vector

- target configuration

Fig. 8 A $90^{\circ}$-corner case for computing a difference vector $\tilde{\boldsymbol{d}}_{k}^{i}$ 
This vector describes the relocation of one design node of the material configuration for the $k$-th iteration step. The vector originates in the spatial configuration and therefore contains only information about the spatial configuration and does not involve information about manufacturing constraints, which potentially limit the external shape of the material configuration. The following approach is the first step to involve manufacturing constraints into the optimization.

Here, the available design space is described by an area (2D). Initially, there is no need to approximate the available design space by nodes and elements. However, we deal with a node-based optimization approach. Furthermore, a comparison between an updated material configuration and an available design space has analogies to the projection-step outlined in the previous section. Figure 9 highlights the way of constraining the update to an available design space. The available design space is approximated by an arbitrary number of nodes. A material nodal position $\boldsymbol{X}_{k}^{i}$ is supposed to be updated with the material difference vector $\boldsymbol{D}_{k}^{i}$. Due to the constraint every updated material nodal position $\boldsymbol{X}_{k+1}^{i}$ is enforced to be inside the available design space. In the case of being outside of the available design space, the node is projected onto the available design space boundary. For this projection, the vector $\hat{\boldsymbol{D}}_{k}^{i}$ is calculated and used to updated the node $\boldsymbol{X}_{k+1}^{i}$ to $\hat{X}_{k+1}^{i}$. In the case a node inside the available design space no further update is calculated.

\section{A two-stage forming process of a tailored blank}

\section{Process description}

The optimization process including the independence of the update on one particular nodal position ("Detachment of the target mesh from the mesh of the forming simulation" section) and the constraint by an available design space ("Update of the material configuration with a limited available design space" section) is demonstrated by an example belonging to the collaborative research project TR73. A two-stage forming process is performed to a circular blank of $2 \mathrm{~mm}$ thickness. Figure 10a shows a cut through the part
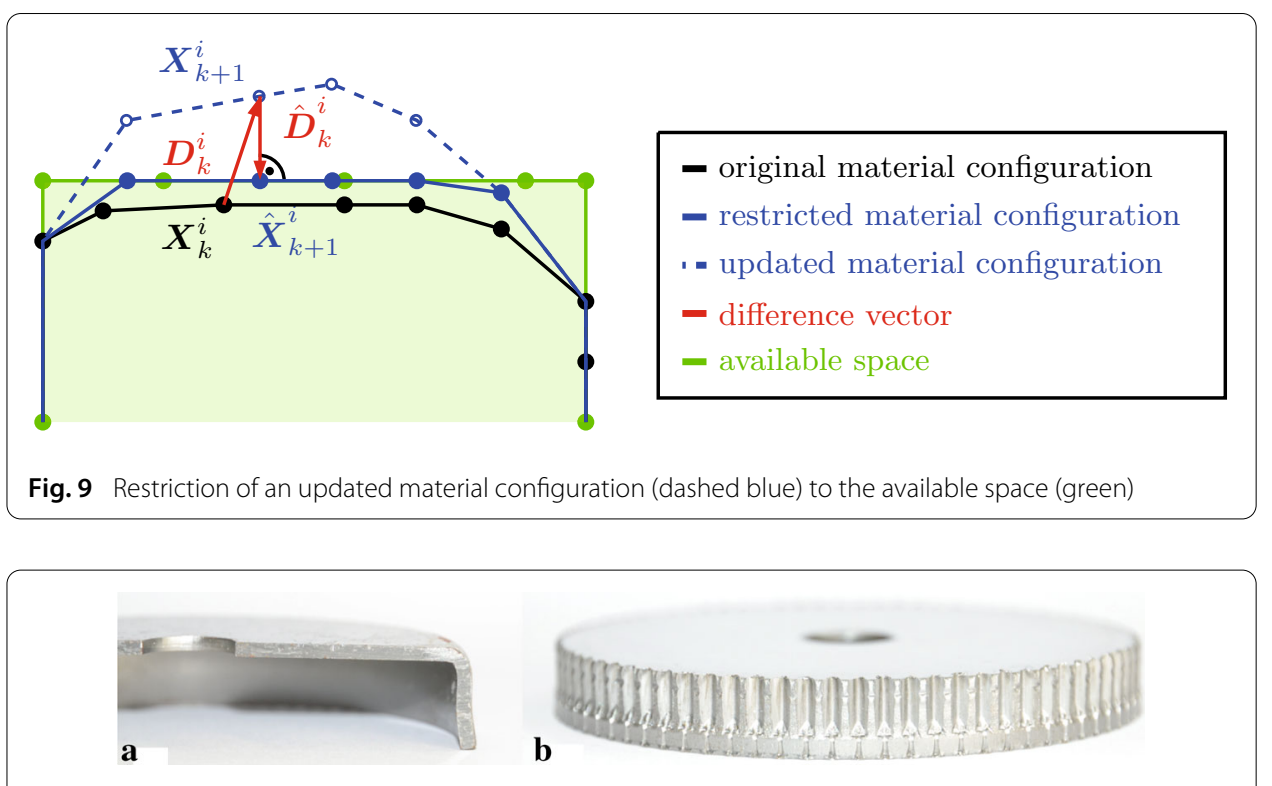

Fig. 10 Demonstrator of the collaborative research project TR73. A cut out $\mathbf{a}$ after the first, $\mathbf{b}$ after the second stage of the forming process 
after the first stage of the combined deep drawing and stamping process. The final shape of the part is displayed in Fig. 10b. The example is a demonstrator that serves for different investigations regarding sheet-bulk metal forming operations [1].

In the following, the forming simulation is reduced to a two dimensional axisymmetric analysis. This dimensional reduction drastically decreases the computational cost. The forming simulation is illustrated in Fig. 11a. The blank measures an internal radius of $7 \mathrm{~mm}$ and an external radius of $50 \mathrm{~mm}$. Combined with the already mentioned thickness, it ends up with a $43 \mathrm{~mm} \times 2 \mathrm{~mm}$ axisymmetric plane. This is approximated by 480 fournoded, isoparametric, quadrilateral elements with a four-point Gaussian integration. The material data of the deep drawing steel DC04 published in [13] is used. The high hardening capacity and low initial yield strength of the DC04 are typical for cold rolled sheet metal and are working well for large deformations. The used material data belong to the HockettSherby model with isotropic hardening Material details are listed in Table 2.

The forming simulation is based on three tools: the upsetting punch p1, the deepdrawing punch $\mathrm{p} 2$ and the deep-drawing ring $\mathrm{p} 3$, which are represented as rigid bodies. During the simulation the upsetting punch moves downwards followed by the deepdrawing ring which bends the circular blank. Between the tools and the blank Coulomb friction with an arctangent approximation is used with a friction coefficient of 0.07. The end of the simulation is highlighted in Fig. 11b, whereby the deep-drawing punch is in the lower position. The green pictured area in Fig. 11c is the area which has to be filled by material after deep-drawing. A $100 \%$ form filling would be the best starting position for

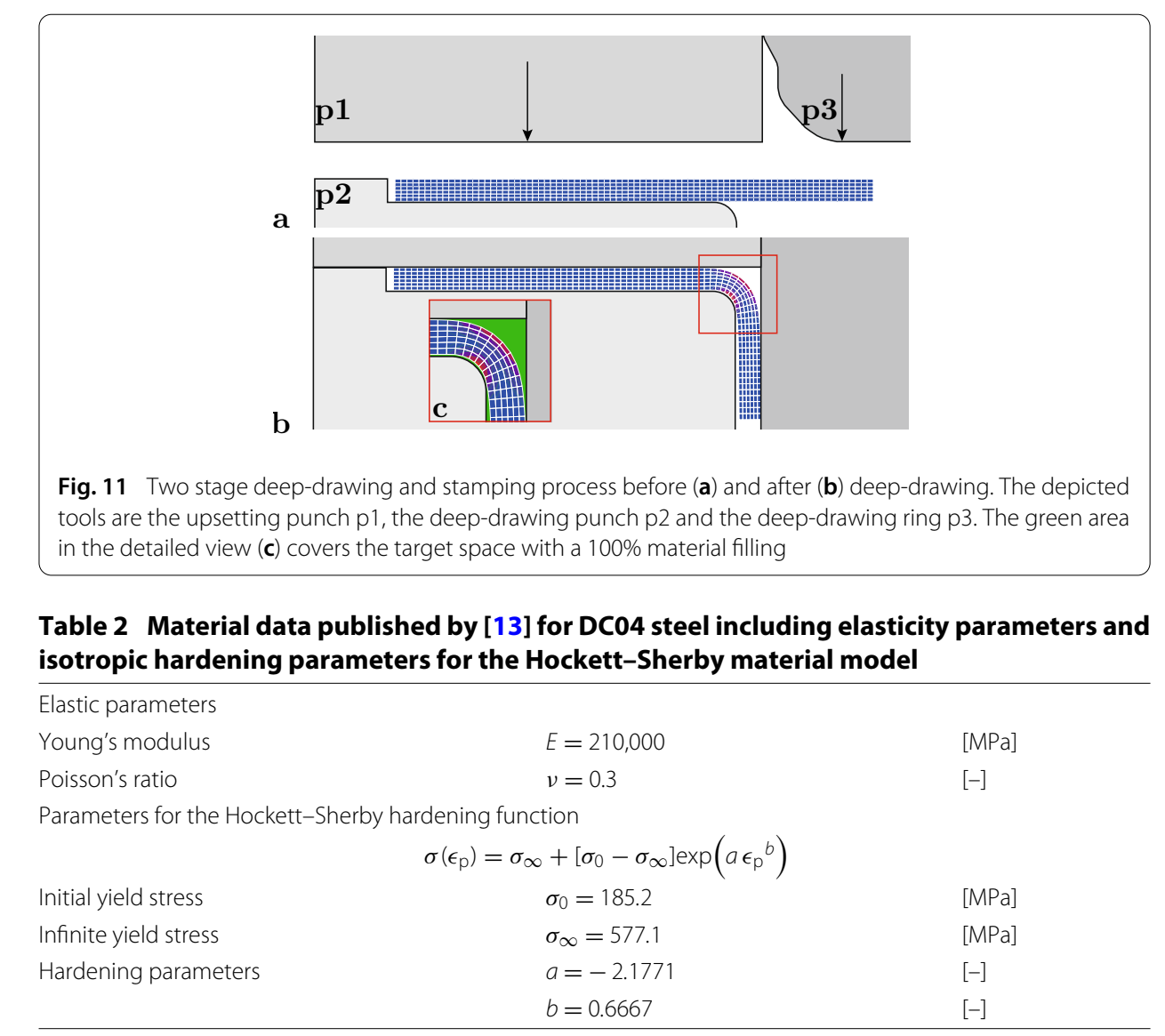




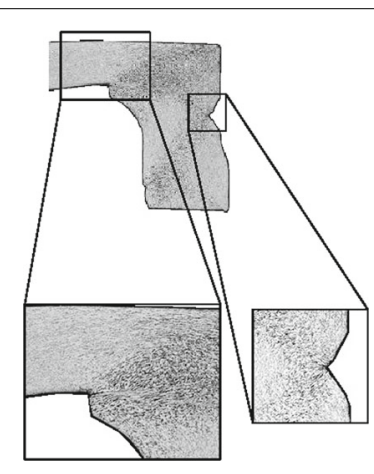

Fig. 12 Experimental observed grain structure of a bended blank after upsetting with the detailed views of two cavity defects [2]

the second stage of the forming process, the upsetting. During the upsetting the teeth are getting impressed into the deep-drawing ring to end up with the demonstrator displayed in Fig. 10. Figure 11b shows the deformed configuration of the forming simulation. The blank is bended by $90^{\circ}$ and the deep-drawing ring is in the lowest position. The detailed view in Fig. 11c shows the challenge of form filling, since the edge is not filled with material. However, for the subsequent second step, the upsetting, it would be useful to have a low material flow towards the edge, which is achieved by already completely filling the edge in the first stage. During the upsetting the material flow has to be concentrated to fill the cavities for the teeth, otherwise those will not be completely filled. The experimentally obtained final configuration in Fig. 12 highlights this form filling defect.

The aim of the optimization is thus to adapt the material configuration, so that the deformed configuration ends up with a better form filling at the corner. For the manufacturing process this optimization is realized by a material pre-distribution step which is part of the overall manufacturing chain. Material pre-distribution is applied either by a rolling operation or an orbital forming operation. Both operations produce an adapted semi-finished product to reach a better form filling. However, the additional height which is generated by the use of pre-distribution is limited and therefore the maximum available design space is restricted. A detailed description of the manufacturing of tailored blanks in sheet-bulk metal forming and the material flow is outlined in [2].

\section{Application of the detached target mesh}

Due to the introduced approach to compute the difference vector ("Detachment of the target mesh from the mesh of the forming simulation" section) it is possible to create a target mesh independently of the mesh for the forming simulation, even though the optimization is node-based. This offers the opportunity to determine the optimal shape and mesh this shape with an arbitrary number of nodes and elements. The applicability of the method is demonstrated by the following example. Figure 13 shows two different target meshes, both represent the same shape. In order to demonstrate the independence of the optimization and the individual positions of a target mesh, the optimal material configuration for the different target meshes is determined. In the case of an equal optimized material configuration the independence from the target mesh is proved. The first target mesh in Fig. 13a consists of 506 nodes and 409 elements, the second (Fig. 13b) of 747 nodes 


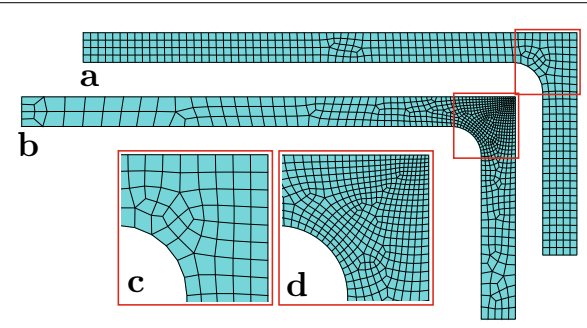

Fig. 13 The two different target configurations (a) and (b) with corresponding detailed views (c) and (d) which are used to evaluate the detachment of the target mesh form the mesh of the forming simulation in "Detachment of the target mesh from the mesh of the forming simulation" section

and 647 elements. The detailed views (Fig. 13c, d) show the significant differences in the area of interest. A further benefit of an independent target mesh is the opportunity to use a high resolution for a complex shape even though the mesh for the forming simulation itself is more coarse.

The optimized material configurations depicted in Fig. 14 result from an optimization by the described process in "Process description" section. The configuration in Fig. 14a results from the first target mesh in Fig. 13a, whereas configuration Fig. 14b is computed with the second target mesh in Fig. 13b. Identical results for both material configurations are obtained, obviously there is no difference between the nodes of the shape which has been updated. The identical result is determined by comparing both deformed configurations in Fig. 15 to their respective material configurations and target meshes. There is no significant difference neither in their total equivalent plastic strains nor in the positions of their nodes.

The optimization results in two identically optimized material configurations and deformed configurations for two different and arbitrarily meshed target configurations.

\section{Constraining the available design space}

The optimization depicted in Fig. 14 is computed without any constraint related to the available design space. The design nodes are updated by usage of Eq. 20 with its nodal difference vector Eq. 21. The constraint of an available design space is outlined in the following. The pre-distribution is applied by using orbital forming or rolling. Due to limits of this forming operations a constraint on the maximum additional blank thickness

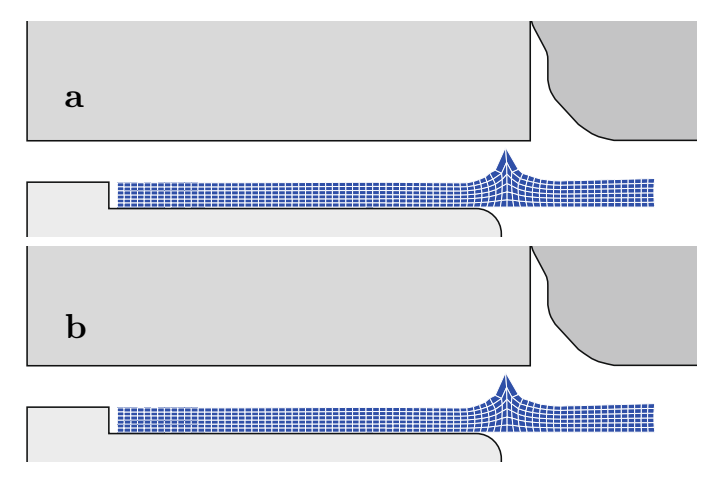

Fig. 14 Optimized material configurations. Configuration a corresponds to the discretized target configuration Fig. 13a, and $\mathbf{b}$ to the discretized target configuration Fig. 13b 


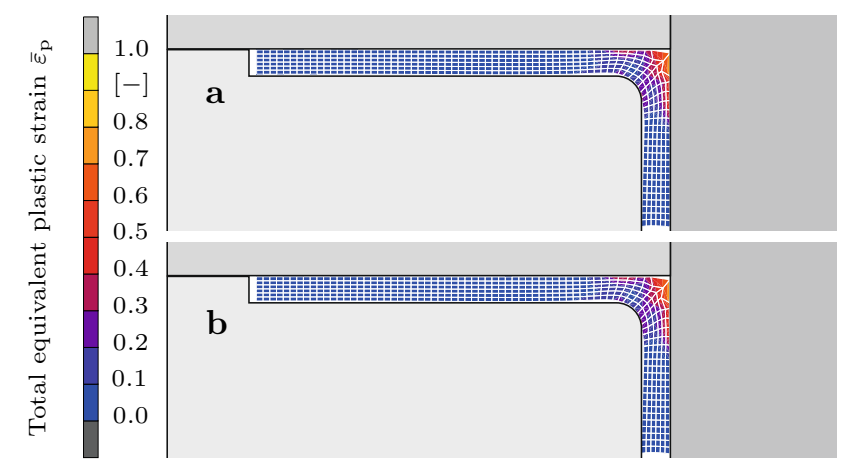

Fig. 15 Spatial (deformed) configurations according to the optimized material configurations. Figure 13a $\rightarrow$ (a), Fig. $13 \mathrm{~b} \rightarrow(\mathbf{b})$

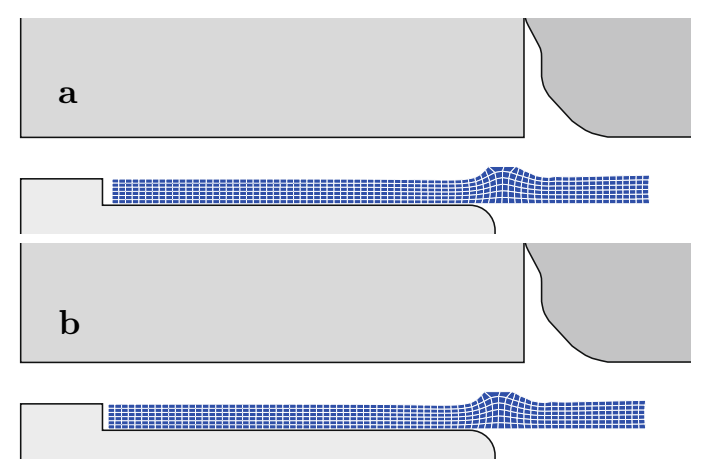

Fig. 16 Optimized material configurations with a constraint of the available design space: configuration a corresponds to the discretized target configuration in Fig. 13a, and $\mathbf{b}$ to the discretized target configuration Fig. $13 b$

produced by an update step is needed. The space is approximated with an arbitrary number of nodes, elements and outlines the shape of the blank with an additional height of $1 \mathrm{~mm}$. The optimization is performed with the same boundary conditions as outlined in the previous chapter. Figure 16 shows the optimized material configurations. Both configurations belong to the same initial material configuration. However, they have different target meshes as already mentioned previously, which does not cause any differences to the optimization result. Constraining the available design space causes a reduced maximum peak of the material pre-distribution. Due to the elastic procedure for updating the interior nodes, also the interior nodes are rearranged in a different way compared to the unconstrained optimization. Limiting the optimization result also causes a limit of the accuracy of the computed spatial configuration. Due to the reduced peak, the form filling in the $90^{\circ}$-corner is reduced compared to the unconstrained results. This side effect is unavoidable, see Fig. 17. However, taking into account the constraint, an optimal shape is still computed by using the advocated form finding approach. The optimization involves a constraint motivated by taking into consideration the manufacturability. The constraint of the available design space is here not specifically adjusted to any realistic conditions. It is a first step towards optimization with forming constraints. 


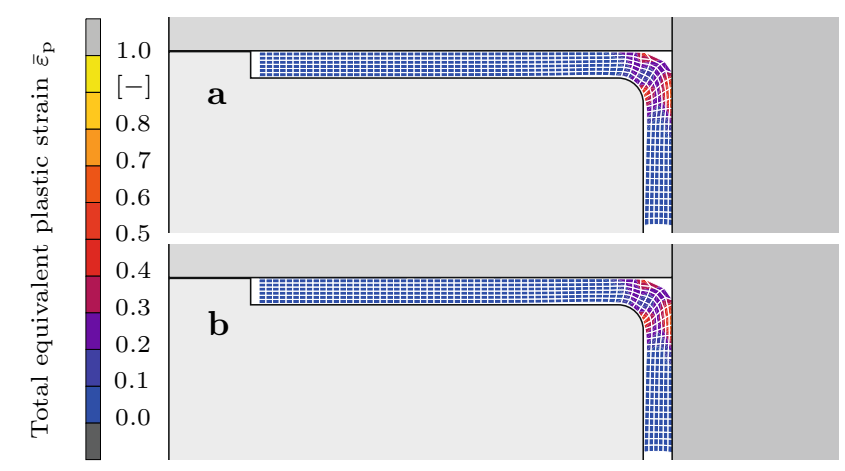

Fig. 17 Spatial (deformed) configurations according to the optimized material configurations with constraint of the available design space. Figure $13 \mathrm{a} \rightarrow(\mathbf{a})$, Fig. 13b $\rightarrow$ (b)

\section{Conclusion}

A form finding approach has been introduced for different forming simulations and is demonstrated to be suited and accurate for a variety of sheet-bulk metal forming applications. Based on the non-invasive character of the optimization approach it is possible to optimize structures of high complexity with nonlinear material behavior, contact constraints and large deformations. The forward simulation is treated within the optimization as a black box. Only input and output files are transferred. Thus, optimization and simulation codes work independently of one another, i.e. in a non-invasive fashion.

Two further innovations are presented. On the one hand a possibility to define the target mesh independently of the mesh for the forming simulation is proposed. One and the same target mesh can be used to optimize discretized structures of different connectivity and element numbers. It is therefore possible to compare different discretizations directly against each other. In addition, it is guaranteed that the result of the optimization, the nodal positions of the material configuration and the corresponding shape, are independent of the position of a single node of the target configuration and are optimized only with regard to its relevant shape. This procedure is verified by comparing the optimization result obtained with two different target discretizations of the same structural analysis.

Furthermore, a first step is presented to perform optimization with regard to the manufacturability of a product. The final material shape may in some cases meet the requirements of the target shape, but there are often shapes that cause production problems. This problem is circumvented if manufacturing constraints are already considered during the optimization. The result of the optimization is therefore already adapted to the production conditions. The presented limitation of the available design space proves to be practicable, although this innovation is only a small step towards production-oriented optimization. The adaption of this procedure to real processes is part of our ongoing investigations.

\section{Authors' contributions}

MC designed the content of the paper and developed the presented innovations based on the code provided by PL. PS supervises the project. All authors have contributed to the preparation of the final manuscript. All authors read and approved the final manuscript. 


\section{Competing interests}

The authors declare that they have no competing interests.

\section{Availability of data and materials}

On request.

Ethics approval and consent to participate

Not applicable.

\section{Consent for publication}

Not applicable.

\section{Funding}

This work is part of the DFG-funded collaborative research project: manufacturing of complex functional components with variants by using a new metal forming process_-sheet-bulk metal forming (SFB/TR73: http://www.tr-73.de).

\section{Publisher's Note}

Springer Nature remains neutral with regard to jurisdictional claims in published maps and institutional affiliations.

Received: 8 January 2018 Accepted: 18 April 2018

Published online: 08 May 2018

\section{References}

1. Merklein M, Allwood JM, Behrens B-A, Brosius A, Hagenah H, Kuzman K, Mori K, Tekkaya AE, Weckenmann A. Bulk forming of sheet metal. CIRP Ann Manuf Technol. 2012;61(2):725-45. https://doi.org/10.1016/j.cirp.2012.05.007.

2. Schulte R, Hildenbrand P, Vogel M, Lechner M, Merklein M. Analysis of fundamental dependencies between manufacturing and processing tailored blanks in sheet-bulk metal forming processes. Procedia Engineering 207(Supplement C), 2017;207:305-10. https://doi.org/10.1016/j.proeng.2017.10.779. In: International conference on the technology of plasticity, ICTP 2017, 17-22 September 2017, Cambridge, United Kingdom.

3. Chenot J-L, Massoni E, Fourment J. Inverse problems in finite element simulation of metal forming processes. Eng Comput. 1996;13(2/3/4):190-225. https://doi.org/10.1108/02644409610114530.

4. Landkammer P, Steinmann P. A non-invasive heuristic approach to shape optimization in forming. Comput Mech. 2016;57(2):169-91. https://doi.org/10.1007/s00466-015-1226-2.

5. Landkammer P, Caspari M, Steinmann P. Improvements on a non-invasive, parameter-free approach to inverse form finding. Computational mechanics. Heidelberg: Springer; 2017. https://doi.org/10.1007/s00466-017-1468-2.

6. Caspar, M, Landkammer P, Steinmann P. Inverse from finding with h-adaptivity and an application to a notch stamping process. In: Computational plasticity XIV on fundamentals and applications. 2017.

7. Steinmann P. Geometrical foundations of continuum mechanics. Heidelberg: Springer; 2015. https://doi.org/10. 1007/978-3-662-46460-1.

8. Altenbach H. Kontinuumsmechanik: einführung in die materialunabhängigen und materialabhängigen gleichungen. Heidelberg: Springer; 2015. https://doi.org/10.1007/978-3-662-47070-1.

9. Wriggers P. Nonlinear finite element methods. Heidelberg: Springer; 2008. https://doi.org/10.1007/ 978-3-540-71001-1.

10. Schmitt O, Friederich J, Riehl S, Steinmann P. On the formulation and implementation of geometric and manufacturing constraints in node-based shape optimization. Struct Multidiscip Optim. 2016;53(4):881-92. https:// doi.org/10.1007/s00158-015-1359-0.

11. Zienkiewicz OC, Zhu JZ. The superconvergent patch recovery and a posteriori error estimates. Part 1 -the recovery technique. Int J Numer Methods Eng. 1992;33(7):1331-64.

12. Sieczkarek P, Wernicke $S$, Gies S, Martins PAF, Tekkaya AE. Incipient and repeatable plastic flow in incremental sheet-bulk forming of gears. Int J Adv Manuf Technol. 2016;86(9--12):3091-100. https://doi.org/10.1007/ s00170-016-8442-6.

13. Schmaltz S, Willner K. Comparison of different biaxial tests for the inverse identification of sheet steel material parameters. Strain. 2014;50(5):389-403. https://doi.org/10.1111/str.12080.STRAIN-0903.R1. 